\title{
Information about Regge-Pole Couplings from Vector-Meson Production*
}

\author{
LORELLA JONES $\dagger$ \\ California Institute of Technology, Pasadena, California \\ (Received 24 April 1967; revised manuscript received 11 August 1967)
}

\begin{abstract}
Experimental density matrices of the vector mesons and isobars produced in reactions of the form $\pi B \rightarrow$ $V B$ and $\pi B \rightarrow V \Delta$ are compared with predictions obtained by assuming particular couplings at the Reggebaryon-baryon vertex. Couplings are classified according to the total angular momentum $J$ (orbital combined with intrinsic) of the Regge trajectory relative to the incident nucleon in the direct channel. For the $\pi B \rightarrow$ $V \Delta$ case, it is found that the present Regge-pole treatment of unequal-mass kinematics allows one to distinguish between couplings for different $J$, and that all data are consistent with $J=1$. Data for $\pi B \rightarrow V B$ charge-exchange reactions are also consistent with $J=1$ dominance.
\end{abstract}

\section{INTRODUCTION}

$\mathrm{R}^{\mathrm{B}}$ EGGE-POLE model analysis of reactions in which several of the external particles have spin is complicated by the large number of independent residue functions (limited only by the requirements of factorization and over-all conservation of parity). Hence it is of great interest to find a model which both fits the data and reduces the number of independent $t$-channel amplitudes. The first step in this direction was taken by Gottfried and Jackson, ${ }^{1}$ who remarked that certain restrictions on couplings due to parity conservation would apply to all exchanged particles with a particular relation between intrinsic parity and spin [e.g., $P=(-1)^{S+1}$ or $\left.P=(-1)^{S}\right]$. Since then, a number of persons have studied the residue functions in various models. $^{2}$ The work most relevant to the discussion here is a static model bootstrap calculation by Dashen and Frautschi, ${ }^{3}$ in which self-consistent couplings of a Regge trajectory and a nucleon to either a nucleon or a $\Delta$ were determined. This model predicted that dipole coupling should dominate the Regge- $N-\Delta$ vertex regardless of the relation between spin and parity for the trajectory; and that spin-flip and non-spin-flip couplings of meson octet trajectories at the Regge- $N-N$ vertex should appear with a certain $S U$ (3) $F$-to- $D$ ratio.

The purpose of this paper is to compare experimental vector-meson and nucleon isobar density matrices with predictions calculated using various Regge-baryonbaryon couplings. Couplings are classified according to the total angular momentum (intrinsic combined with orbital) of the exchanged object relative to the incident nucleon in the $s$ channel. An approximate form of the helicity amplitude crossing relations is used to determine the restrictions placed by a particular coupling on the $t$-channel helicity amplitudes; the resulting $t$-channel

\footnotetext{
* Work supported in part by the U. S. Atomic Energy Commis-

${ }^{*}$ Work supported in part by the U. S. Atomic Energy Commis-
sion. Prepared under Contract No. AT(11-1)-68 for the San Francisco Operations Office, U. S. Atomic Energy Commission.

$\dagger$ National Science Foundation Predoctoral Fellow.

1 K. Gottfried and J. D. Jackson, Nuovo Cimento 33, 309 (1964).

${ }^{2}$ Y. Hara, Phys. Rev. 140, B178 (1965); 140, B1649 (1965).

${ }^{3}$ R. Dashen and S. C. Frautschi, Phys. Rev. 152, 1450 (1966).
}

amplitudes are then used to compute the density matrix elements.

It is found that magnetic dipole coupling at the Regge- $N-\Delta$ vertex requires $\left(\rho_{33}+\sqrt{3} \rho_{3-1}\right) /\left(\rho_{11}+\rho_{1-1}\right)=\frac{3}{4}$ $\left(\rho_{33}, \rho_{3-1}\right.$, and $\rho_{31}$ are the easily measured elements of the $\Delta$ density matrix; $\rho_{11}, \rho_{00}, \rho_{1-1}$, and $\rho_{10}$ are elements of the $V$ density matrix), and places no restriction on

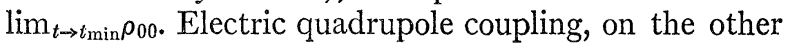
hand, requires $\lim _{t \rightarrow t_{\min } \rho_{00}}=0$, but does not specify any simple linear relation between the $V$ and $\Delta$ densitymatrix elements. These predictions are independent of the parity exchanged. Examination of the data for these processes indicates that $\rho_{00}$ does not approach 0 at low $t$, and that $\left(\rho_{33}+\sqrt{3} \rho_{3-1}\right) /\left(\rho_{11}+\rho_{1-1}\right)=\frac{3}{4}$ is consistent with the range of $(0.6,0.8)$ defined by experiment; these facts support a picture of predominantly dipole coupling. For those reactions in which $P=(-1)^{S+1}$ exchange is expected to dominate (production of $\rho$ and $K^{*}$ ), the experimental value $\lim _{t \rightarrow i_{\min } \rho_{00}} \approx 0.8$ indicates that quadrupole coupling contributes at most $\frac{1}{5}$ of the cross section at $t_{\mathrm{min}}$; dipole dominance for $P=(-1)^{S}$ exchanges is consistent with all the data for $\pi N \rightarrow \pi \Delta$. Hence the data for both parities individually agrees with the Dashen-Frautschi prediction.

For octet trajectories, the Dashen-Frautschi calculation predicts that the ratio of couplings $g^{\operatorname{Regge}-N-\Delta} /$ $g^{\text {Regge }-N-N}$ for $J=1$ vertices should have a particular value. It also predicts that the $\rho N N$ and $A_{2} N N J=0$ (non-spin-flip) couplings will be small. A calculation incorporating only these predictions [(a) both Regge$N-\Delta$ and Regge- $N-N$ charge-exchange vertices are dominated by $J=1$; and (b) the Regge- $N-\Delta$ and Regge- $N-N$ vertices have the predicted ratio] leads to the relation $\rho_{i j}=\rho_{i j}{ }^{\prime}$, where $\rho_{i j}$ is the vector-meson density matrix in charge-exchange $\pi B \rightarrow V B$, and $\rho_{i j}{ }^{\prime}$ is the vector-meson density matrix for the comparable $\pi B \rightarrow V \Delta$ reaction. This relation is also well satisfied experimentally.

The approximations made in this calculation are such that predictions of limits at $t \rightarrow t_{\min }$ and shapes of density matrix elements near this point should be taken more seriously than average values. The agreement with experimental data averaged over a larger range of $t$ is, however, impressive. 


\section{METHOD}

For purposes of comparison with other authors, it is most convenient to define couplings in the rest system of one of the particles involved. Consider the coupling of a meson trajectory with an incident baryon to produce a final baryon. In order to couple to a spin- $\frac{1}{2}$ particle at rest, the total angular momentum $(\mathbf{J}=\mathbf{S}+\mathbf{L})$ of the Reggeon relative to the incident nucleon must be $0^{+}$ or $1^{+}$.

As examples of relativistic couplings with this character which are nonvanishing in the static limit, consider

$$
\begin{array}{r}
\bar{N}_{2} N_{1} \sim X^{+} \phi\left[1-\frac{p_{2}{ }^{2}}{\left(E_{1}+M\right)\left(E_{2}+M\right)}\right] \\
-\frac{X^{+} \boldsymbol{\sigma} \cdot \mathbf{p}_{2} \boldsymbol{\sigma} \cdot\left(\mathbf{p}_{1}-\mathbf{p}_{2}\right) \phi}{\left(E_{1}+M\right)\left(E_{2}+M\right)}, \\
\bar{N}_{2} \gamma_{\mu} \gamma_{5} N \sim\left(A_{0}, \mathbf{A}\right), \\
A_{0}=X^{+} \boldsymbol{\sigma} \phi \cdot\left[\frac{\mathbf{p}_{1}}{E_{1}+M}+\frac{\mathbf{p}_{2}}{E_{2}+M}\right], \\
\mathbf{A}=X^{+} \boldsymbol{\sigma} \phi\left[1-\frac{p_{2}{ }^{2}}{\left(E_{1}+M\right)\left(E_{2}+M\right)}\right] \\
+\frac{2 \mathbf{p}_{2} \mathbf{p}_{2} \cdot X^{+} \boldsymbol{\sigma} \phi}{\left(E_{1}+M\right)\left(E_{2}+M\right)} \\
+\frac{X^{+} \boldsymbol{\sigma} \cdot \mathbf{p}_{2} \boldsymbol{\sigma \sigma} \cdot\left(\mathbf{p}_{1}-\mathbf{p}_{2}\right) \phi}{\left(E_{1}+M\right)\left(E_{2}+M\right)}
\end{array}
$$

where $X$ and $\phi$ are two-component spinors. At $\mathbf{p}_{2}=0$, coupling (a) between the two spinors is purely of type $X^{+} \phi\left(0^{+}\right)$and coupling (b) is purely of type $X^{+} \boldsymbol{\sigma} \phi\left(1^{+}\right)$. For $\mathbf{p}_{2} \neq 0$, admixtures of other types of couplings are proportional to $\mathbf{p}_{1}-\mathbf{p}_{2}$. Hence if the momentum transfer between the nucleons $\left(\mathbf{p}_{1}-\mathbf{p}_{2}\right)$ remains small, the $J$ character of the coupling (whether it is $0^{+}$or $1^{+}$) is independent of the system of coordinates.

Similar results can be obtained for the coupling of a meson trajectory with a nucleon to yield a $\Delta\left(J_{\Delta}=\frac{3}{2}\right)$. Here the $1^{+}$(dipole) coupling is obtained, for example, from (a) by allowing $N_{2}$ to be one component of the four-vector $\Delta_{\nu}$; the $2^{+}$(quadrupole) coupling may be obtained from (b) by the same substitution.

These examples indicate that if a trajectory couples principally by a particular type of coupling (scalar, dipole, or quadrupole) in the static limit $\mathbf{p}_{1} / M=\mathbf{p}_{2} / M$ $=0$, then it should have roughly the same type of coupling at small $t$ regardless of the energies of the nucleons involved. This allows us to work in the $s$ channel center-of-mass system for high energy, while comparing our results with those of computations performed by Dashen and Frautschi in a static model.

Classification of couplings by $J$ allows us to find relations between different amplitudes containing the same coupling. For example, in an amplitude of the form $f=X^{+} \boldsymbol{\sigma} \phi \cdot V$ ( $X$ and $\phi$ are two-component nucleon spinors), there is the definite relation $f_{++}=V_{z}=-f_{--}$. In order to generalize these equations for different $J$ 's and different processes, it is convenient to notice the following fact: Provided the momentum of the new baryon is collinear with that of the incident nucleon, the ratio of two helicity amplitudes with the same change of baryon helicity is given by the ratio of Clebsch-Gordan coefficients for coupling to the nucleon. For example,

$$
\frac{f_{c_{\frac{3}{2} ;} ; 0^{\frac{1}{2}}}}{f_{c^{\frac{1}{2}} ; 0-\frac{1}{2}}{ }^{s}}=\frac{\left(J \frac{1}{2} 1 \frac{1}{2} \mid \frac{3}{2} \frac{3}{2}\right)}{\left(J \frac{1}{2} 1-\frac{1}{2} \mid \frac{3}{2} \frac{1}{2}\right)},
$$

if the Reggeon couples with total angular momentum $J$. These relations are exact only at $\theta_{s}=0^{\circ}$. In order to simplify calculations, they will be assumed to hold over a range of small $t$ in the $s$-channel center-of-mass system.

As in any Regge-pole model, it is most convenient to express the differential cross sections and density matrix elements in terms of $t$-channel helicity amplitudes. These are related to the $s$-channel amplitudes by the Trueman-Wick crossing relations

$$
\begin{aligned}
f_{c 0 ; a b} t(s, t)=\sum_{A^{\prime}, b^{\prime}, c^{\prime}} & d_{A^{\prime} a}^{J a}\left(X_{a}\right) \\
& \times d_{b^{\prime} b^{1 / 2}}\left(X_{b}\right) d_{c^{\prime} c}\left(X_{c}\right) f_{c^{\prime} A^{\prime} ; 0 b^{\prime}},
\end{aligned}
$$

where indices $b$ and $b^{\prime}$ refer to the incident nucleon, $a$ and $A^{\prime}$ to the final baryon, $c$ and $c^{\prime}$ to the vector meson, and $d=d^{\prime}=0$ to the pion, and

$$
\begin{aligned}
\sin X_{a}= & \frac{2 m_{a} \sqrt{ } \phi(s, t)}{\tau_{a b} s_{a c}}, \\
\sin X_{b}= & \frac{2 m_{b} \sqrt{ } \phi(s, t)}{\tau_{a b} s_{b d}}, \quad \sin X_{c}=\frac{2 m_{c} \sqrt{ } \phi(s, t)}{\tau_{c d} s_{a c}}, \\
\tau_{i j}= & {\left[t-\left(m_{i}+m_{j}\right)^{2}\right]^{1 / 2}\left[t-\left(m_{i}-m_{j}\right)^{2}\right]^{1 / 2}, } \\
s_{i j}= & {\left[s-\left(m_{i}+m_{j}\right)^{2}\right]^{1 / 2}\left[s-\left(m_{i}-m_{j}\right)^{2}\right]^{1 / 2}, } \\
\phi(s, t)= & s t\left(\sum m_{i}{ }^{2}-s-t\right)-t\left(m_{b}{ }^{2}-m_{d}{ }^{2}\right)\left(m_{a}{ }^{2}-m_{c}{ }^{2}\right) \\
& -s\left(m_{a}{ }^{2}-m_{b}{ }^{2}\right)\left(m_{c}{ }^{2}-m_{d}{ }^{2}\right) \\
& -\left(m_{a}{ }^{2} m_{d}{ }^{2}-m_{c}{ }^{2} m_{b}{ }^{2}\right)\left(m_{a}{ }^{2}+m_{d}{ }^{2}-m_{c}{ }^{2}-m_{b}{ }^{2}\right),
\end{aligned}
$$

where $\phi(s, t)=0$ is the boundary of the physical region.

Equation (3) as it stands is rather complicated and cumbersome to apply. To simplify the form of conditions on $t$-channel amplitudes caused by the coupling relations, we make a number of approximations based on the fact that the results derived are to be tested at high energies and very close to the forward direction.

For the reaction $\pi B \rightarrow V B, E q$. (4) shows that $X_{a}(t) \approx X_{b}(t)$ at large $s$. To the extent that $X_{a}(t)$ $=X_{b}(t)$, the $s$-channel relations for scalar coupling $\left(f_{c \frac{1}{3} ; 0 \frac{1}{3}^{8}}=f_{c-\frac{1}{3} ; 0-\frac{2^{8}}{2}} ; f_{c} ; 0-\frac{2^{8}}{2}=0=f_{c-\frac{1}{2} ; 0 \frac{1}{3}^{8}}\right)$ imply simi- 
TABLE I. Predictions of the model for reactions of the form $\pi B \rightarrow V B$.

(1) Scalar coupling at the Regge- $N-N$ vertex. This coupling is allowed only for $P=(-1)^{S}$ exchanges.

(a) Restrictions due to coupling:

$$
\begin{gathered}
f_{c 0 ;-\frac{1}{2} \frac{1}{2}} t=0=f_{c 0 ; \frac{1}{2}-\frac{1}{3}} t, \\
f_{c 0 ;-\frac{1}{2}-\frac{1}{2}} t=f_{\left.c 0 ; \frac{1}{3}\right)^{t}} .
\end{gathered}
$$

(b) Restrictions due to parity:

$$
f_{00 ; a b}{ }^{t}=0 \text {. }
$$

(c) Predicted vector-meson density matrix:

$$
\rho_{00}=0=\rho_{10} ; \quad \rho_{11}=\frac{1}{2}=\rho_{1-1} .
$$

(d) Predicted cross-section shape at low $t$ :

$$
d \sigma / d t \sim \sin ^{2} \theta_{t} .
$$

(2) Dipole coupling at Regge- $N-N$ vertex.

(a) Restrictions on $t$-channel amplitudes due to coupling:

$$
f_{c 0 ; \frac{1}{2} \frac{1}{3}} t=-f_{c 0 ;-\frac{1}{2}-\frac{1}{2} t} \text {. }
$$

(b) Further predictions if only $P=(-1)^{S}$ is exchanged:

$$
\text { Parity } \Rightarrow f_{00 ; a b}{ }^{t}=0 \text {, }
$$

$$
\text { Coupling + parity } \Rightarrow f_{10 ; \frac{1}{2} \frac{1}{3}} t=0,
$$

Vector-meson density matrix:

$$
\rho_{00}=0=\rho_{10} ; \quad \rho_{11}=\frac{1}{2} ; \quad \rho_{1-1}=\frac{1}{2}\left[\frac{\cos ^{2} \theta_{t}-1}{\cos ^{2} \theta_{t}+1}\right] .
$$

(c) Further predictions if only $P=(-1)^{S+1}$ is exchanged Kinematical restrictions only (Ref. 6).

lar relations for the $t$-channel amplitudes. Likewise, the conditions for dipole coupling are true in either channel. Hence, despite the very rapid change in nucleon crossing angles near $\theta_{s}=0^{\circ}$, the relations listed in Table I depend only on the assumption of collinearity made in Eq. (2), and the energy involved.

An additional approximation is necessary for treatment of $\pi B \rightarrow V \Delta$. In this reaction, the baryon crossing angles vary considerably more slowly near $\theta_{s}=0^{\circ}$ than for the case of $\pi B \rightarrow V B$. $\operatorname{Sin} X_{a}$ and $\sin X_{b}$ also vary much more slowly in the extreme forward direction than $\sin \theta_{t}=(t \phi)^{1 / 2} / \tau_{a b} \tau_{c d}\left(\sin \theta_{t}\right.$ gives an appropriately dimensioned measurement of the variation of densitymatrix elements). If we replace these relatively slowly varying portions of the crossing matrix by their values at $t=t_{\min }$, Eq. (3) may be written as

$$
f_{c 0 ; a b}{ }^{t}=\sum d^{1}{ }_{c^{\prime} c}\left(X_{c}\right) f_{c^{\prime} a ; 0 b^{8}}
$$

near the forward direction. Our coupling conditions on $f_{c^{\prime} a ; 0 b}{ }^{s}$ are independent of $c^{\prime}$ [viz. Eq. (2)] and hence may be translated directly into conditions on $f_{c 0 ; a b}{ }^{t}$ (e.g., the relation $f_{c^{\prime} \frac{1}{2} ; 0 \frac{1}{2}}^{s}=-f_{c^{\prime}-\frac{1}{2} ; 0-\frac{1}{2}}{ }^{s}$ implies $f_{c 0 ; \frac{1}{2} \frac{1}{2}}{ }^{t}$ $\left.=-f_{c 0 ;-\frac{1}{2}-\frac{1}{2}} t\right)$. Hence, near the forward direction, this set of approximations provides in a very direct way relationships between $t$-channel amplitudes corresponding to a given $s$-channel coupling. ${ }^{4}$

${ }^{4}$ These relations (when combined with parity restrictions) check with previously well-known facts. Pursuance of the steps outlined shows, for example, that assumption of $J=1$ Regge-baryon-baryon coupling results in: (a) spin-flip dominance of $\pi N$ charge-exchange
Further restrictions are imposed on the $t$-channel helicity amplitudes by: (i) over-all conservation of parity, which gives

$$
f_{c 0 ; a b} t=(-1)(-1)^{1 / 2-J a}(-1)^{a-a+b} f_{-c 0 ;-a-b}{ }^{t} ;
$$

and (ii) specification of definite spin parity $P=(-1)^{S}$ or $P=(-1)^{S+1}$ for the exchanged trajectory. Exchange of a definite spin parity requires $T_{c 0 ; a b}{ }^{j}=P(-1)^{S+1} T_{-c 0 ; a b}{ }^{j}$ for the partial-wave amplitude $T^{j}$ defined by $f_{c 0, a b} t$

TABLE II. Predictions of the model for reactions of the form $\pi B \rightarrow V \Delta$.

(1) Dipole coupling at the Regge- $N-\Delta$ vertex.

(a) Restrictions on $t$-channel amplitudes due to coupling:

$$
\begin{aligned}
& f_{c 0 ;-\frac{1}{2} 3^{t}}=0=f_{c 0 ; \frac{1}{2}-\frac{1}{3}}{ }^{t}, \\
& f_{c 0 ; \frac{1}{3}} t=\sqrt{3} f_{c 0 ; \frac{1}{3}-\frac{1}{2}}{ }^{t}, \\
& f_{c 0 ;-\frac{1}{2}-\frac{1}{2}}{ }^{t}=f_{c 0 ; \frac{1}{2} \frac{1}{2}^{t}} \text {, } \\
& f_{c 0 ;-1-\frac{1}{1}} t=\sqrt{3} f_{c 0 ;-\frac{1}{2} \frac{1}{2}}{ }^{t} \text {. }
\end{aligned}
$$

(b) General effects of dipole coupling:

General formulas for the density matrix elements (any mixture of parities exchanged) satisfy $\left(\rho_{33}+\sqrt{3} \rho_{3-1}\right) /$ $\left(\rho_{11}+\rho_{1-1}\right)=\frac{3}{4}$ where $\rho_{33}$ and $\rho_{3-1}$ are elements of the $\Delta$ density matrix and $\rho_{11}, \rho_{1-1}$ belong to the vector-meson density matrix.

(c) Further predictions if only $P=(-1)^{S}$ is exchanged:

$$
\text { Parity } \Rightarrow f_{00 ; a b}{ }^{t}=0 \text {, }
$$$$
\text { Coupling + parity } \Rightarrow f_{10 ; \frac{1}{2} \frac{1}{3}} t=0 \text {, }
$$

Vector-meson density matrix:

$$
\rho_{00}=0=\rho_{10} ; \quad \rho_{11}=\frac{1}{2} ; \quad \rho_{1-1}=\frac{\left(\cos ^{2} \theta_{t}-1\right)}{2\left(\cos ^{2} \theta_{t}+1\right)},
$$

$\Delta$ density matrix:

$$
\rho_{31}=0 ; \quad \rho_{33}=\frac{3}{8} ; \quad \rho_{3-1}=\frac{\sqrt{3}}{8} \frac{\left(\cos ^{2} \theta_{t}-1\right)}{\left(\cos ^{2} \theta_{t}+1\right)} .
$$

(d) Further predictions if only $P=(-1)^{S+1}$ is exchanged: Kinematical restrictions only (Ref. 6).

(2) Quadrupole coupling at the Regge- $N-\Delta$ vertex.

(a) Restrictions on $t$-channel amplitudes due to coupling:

$$
\begin{gathered}
f_{c 0 ; 1 \frac{1}{3}} t=-\frac{1}{\sqrt{3}} f_{c 0 ; \frac{1}{2}-\frac{1}{2}} t \\
f_{c 0 ; \frac{1}{2} \frac{1}{3}^{t}}=-f_{c 0 ;-\frac{1}{2}-\frac{1}{2}}{ }^{t}, \\
f_{c 0 ;-\frac{1}{2}-\frac{1}{2}} t=-\frac{1}{\sqrt{3}} f_{c 0 ;-\frac{1}{2} \frac{1}{2}^{t} .}
\end{gathered}
$$

(b) General effects of quadrupole coupling:

Parity+coupling $\Rightarrow f_{00 ; \frac{1}{3}}{ }^{t}=0$.

(c) Further predictions if only $P=(-1)^{S}$ is exchanged: Kinematical restrictions only (Ref. 6 ).

(d) Further predictions if only $P=(-1)^{S+1}$ is exchanged:

Near $t_{\min }, \rho_{00} \sim\left(\sin ^{2} \theta_{t}\right) / t$.

scattering, and (b) the well-known Stodolsky-Sakurai results for $\pi N \rightarrow \pi \Delta$.

For energies in the range 4-8 GeV/c, however, the angles $X_{a-\Delta}$ and $X_{b=N}$ in $\pi N \rightarrow V \Delta$ can be considered small $\left(\Sigma 30^{\circ}\right.$ ) only in the range $\left(t_{\min }, t_{\min }-0.05\right) \mathrm{GeV}^{2}$. Thus the agreement found below between predictions for dipole coupling using Eq. (5) and experiments averaged over a much larger range of $t$ is somewhat surprising.

${ }_{5}^{5}$ M. Jacob and G. C. Wick, Ann. Phys. (N. Y.) 7, 404 (1959). 
TABLE III. Experimental information on $\pi B \rightarrow V \Delta$ density matrices. The range of $R$ consistent with all limits is $0.6 \leq\left(\rho_{33}+\sqrt{3} \rho_{3-1}\right) /\left(\rho_{11}+\rho_{1-1}\right) \leq 0.8$.

\begin{tabular}{|c|c|c|c|c|c|c|}
\hline Experiment & $\rho_{33}$ & $\rho_{3-1}$ & $\lim _{t \rightarrow 0} \rho_{00}$ & $\rho_{11}$ & $\rho_{1-1}$ & $R=\left(\rho_{33}+\sqrt{3} \rho_{3-1}\right) /\left(\rho_{11}+\rho_{1-1}\right)$ \\
\hline$\pi^{+} p \rightarrow \rho^{0} N^{*++3}$ & & & & & & \\
\hline $8 \mathrm{GeV} / c$ & $0.05 \pm 0.03$ & $0.015 \pm 0.028$ & $\sim 0.8$ & $0.12 \pm 0.03$ & $-0.035 \pm 0.024$ & $0.895_{-0.9}+4.0$ \\
\hline$\underset{\substack{+\infty \\
\tau^{+} \rightarrow \rightarrow \omega^{0} N^{*++a}}}{ }$ & $0.08 \pm 0.03$ & $0.01 \pm 0.03$ & $\sim 0.8$ & $0.12 \pm 0.03$ & $-0.04 \pm 0.03$ & $1.25_{-1.1}^{+7.8}$ \\
\hline $8 \mathrm{GeV} / c$ & $0.24 \pm 0.08$ & $0.017 \pm 0.085$ & & $0.37 \pm 0.05$ & $0.17 \pm 0.08$ & $0.5_{-0.4}+0.7$ \\
\hline $4 \mathrm{GeV} / c$ & $0.15 \pm 0.04$ & $0.04 \pm 0.04$ & & $0.27 \pm 0.03$ & $0.13 \pm 0.05$ & $0.55_{-0.32}+0.45$ \\
\hline $\begin{array}{c}\pi^{-} p \rightarrow \omega N^{0 * \mathrm{~b}} \\
2.75 \mathrm{GeV} / c \\
\pi^{+} p \rightarrow \omega N^{*++b}\end{array}$ & $0.18 \pm 0.05$ & $-0.02 \pm 0.05$ & & $0.28 \pm 0.05$ & $0.19 \pm 0.07$ & $0.32_{-0.5}^{+0.5}$ \\
\hline $\begin{array}{c}2.75 \mathrm{GeV} / c \\
K^{+} p \rightarrow K^{*} N^{* c}\end{array}$ & $0.15 \pm 0.01$ & $0.03 \pm 0.01$ & & $0.22 \pm 0.01$ & $0.02 \pm 0.02$ & $0.83_{-0.2^{+0.3}}$ \\
\hline $3.5 \mathrm{GeV} / c$ & & & $\sim 0.75$ & & & \\
\hline$\Delta^{2}=0.1 \mathrm{GeV}^{2}$ & $0.12 \pm 0.1$ & $-0.1 \pm 0.07$ & & $0.15 \pm 0.06$ & $-0.13 \pm 0.08$ & $-2.5_{-\infty}^{+\infty}$ \\
\hline 0.15 & $0.03 \pm 0.1$ & $0 \quad \pm 0.07$ & & $0.13 \pm 0.06$ & $0 \quad \pm 0.06$ & $0.23_{-19}+25$ \\
\hline 0.23 & $0 \pm 0.1$ & $-0.5 \pm 0.07$ & & $0.14 \pm 0.06$ & $0.03 \pm 0.08$ & $-0.53_{-10^{+3.8}}$ \\
\hline 0.63 & $0 \pm 0.1$ & $-0.6 \pm 0.07$ & & $0.18 \pm 0.06$ & $0.1 \pm 0.07$ & $-0.37_{-1.8}^{+1.2}$ \\
\hline $\begin{array}{c}K^{+} p \rightarrow K^{*} N^{* \mathrm{c}} \\
5 \mathrm{GeV} / c\end{array}$ & & & $\sim 0.9$ & & & \\
\hline$\Delta^{2}=0.08 \mathrm{GeV}^{2}$ & $0.2 \pm 0.08$ & $0.03 \pm 0.06$ & & $0.09 \pm 0.08$ & $0.06 \pm 0.06$ & $1.67_{-1.4}^{+42}$ \\
\hline 0.18 & $0.2 \pm 0.1$ & $-0.03 \pm 0.1$ & & $0.19 \pm 0.08$ & $-0.1 \pm 0.08$ & $1.66_{-\infty}^{+\infty}$ \\
\hline 0.4 & $0.2 \pm 0.1$ & $0.03 \pm 0.08$ & & $0.19 \pm 0.1$ & $0.08 \pm 0.08$ & $0.93_{-0.9}{ }^{+4.5}$ \\
\hline
\end{tabular}

a Aachen-Berlin-CERN collaboration, Phys. Letters 22, 533 (1966). b Saclay-Orsay-Bari-Bologna collaboration, Phys. Letters 21, 354 (1966).

$=\sum_{j} d_{a-b, c}{ }^{j}\left(\theta_{t}\right) T_{c 0, a b^{j}}$. The combination of parity restrictions (i) and (ii) with the restrictions imposed by $s$-channel coupling serves to eliminate further amplitudes. Those $t$-channel helicity amplitudes which survive are then inserted into the density matrix formula ${ }^{1}$

$$
\rho_{i j}=\sum g_{\lambda}{ }^{i} g_{\lambda}{ }^{j *} / \sum\left|g_{\lambda}{ }^{k}\right|^{2},
$$

where the sum is over all sets $\lambda$ of helicities for particles other than the one under consideration.

Conditions due to couplings and parity conservation are listed in Tables I and II for the reactions under consideration. Also listed are the values of $\rho_{i j}$ obtained in cases where the model gives a definite prediction. In the remaining cases, only predictions due to unequalmass kinematics can be made. These have been discussed. ${ }^{6}$

\section{DISCUSSION}

$$
\pi N \rightarrow V \Delta \text { (Tables II and III) }
$$

Regardless of the combination of trajectories exchanged or their parities, our set of approximations leads to the requirement for dipole coupling that

$$
R=\left(\rho_{33}+\sqrt{3} \rho_{3-1}\right) /\left(\rho_{11}+\rho_{1-1}\right)=\frac{3}{4},
$$

where $\rho_{33}$ and $\rho_{3-1}$ are elements of the $\Delta$ density matrix and $\rho_{11}, \rho_{1-1}$ belong to the vector-meson density matrix. It is possible that quadrupole coupling might also produce this value for $R$. However, because quadrupole coupling requires $f_{00, \frac{1}{2} \frac{1}{2}} t=0$, it imposes on the vectormeson density matrix the behavior $\rho_{00} \sim \sin ^{2} \theta_{t} / t \sim \phi$ near $\phi=0 .{ }^{6}$ Thus a large value of $\rho_{00}$ at $t_{\mathrm{min}}$ implies that dipole coupling is important; this can then be checked by computing $R .^{7}$

${ }^{6}$ Lorella Jones, preceding paper, Phys. Rev. 163, 1523 (1967).

${ }^{7} P=(-1)^{S}$ exchanges cannot contribute to $\rho_{00}$; hence if only
The available data for these reactions are listed in Table III. They have the following properties:

(i) $\rho_{00}$ certainly does not $\rightarrow 0$ as $t \rightarrow t_{\min }$ for $\rho$ and $K^{*}$ production.

(ii) $R=\left(\rho_{33}+\sqrt{3} \rho_{3-1}\right) /\left(\rho_{11}+\rho_{1-1}\right)=0.75$ is consistent

\begin{tabular}{|c|c|c|}
\hline (1) & Dipole coupling. & Predictions \\
\hline \multicolumn{3}{|c|}{ Restrictions due to coupling and parity: } \\
\hline \multicolumn{3}{|c|}{$f_{00 ;-\frac{3}{2} \frac{1}{2}} t=0=f_{00 ; \frac{1}{2}-\frac{1}{2} t} t$} \\
\hline \multicolumn{3}{|c|}{$f_{00 ;-\frac{1}{2}-\frac{1}{2}} t=0=f_{00 ; \frac{1}{2} \frac{1}{2}}$} \\
\hline
\end{tabular}
with all the reactions (in fact, if one believes the

TABLE IV. Predictions and data for $\pi^{+} p \rightarrow \pi^{0} \Delta^{++}$.

These lead to the Stodolsky-Sakurai results ${ }^{\mathrm{a}}$ for the $\Delta$ density matrix:

$$
\rho_{33}=\frac{3}{8} ; \quad \rho_{31}=0 ; \quad \rho_{3-1}=\sqrt{3} / 8 .
$$

(2) Quadrupole coupling.

Restrictions due to coupling and parity:

$$
\begin{aligned}
& f_{00 ; \frac{1}{3}} t=-\frac{1}{\sqrt{3}} f_{00 ; \frac{1}{2}-\frac{1}{2}} t=-\frac{1}{\sqrt{3}} f_{00 ;-\frac{1}{2}} \frac{1}{2}^{t}=j 00 ;-\frac{1}{2}-\frac{1}{2} t, \\
& f_{00 ; \frac{1}{2}} t=-f_{00 ;-\frac{1}{2}-\frac{1}{2} t} \text {. }
\end{aligned}
$$

No predictions can be made.

$$
\text { Data }
$$

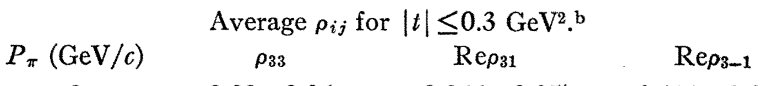

$\begin{array}{llrl}8 & 0.22 \pm 0.06 & 0.066 \pm 0.057 & 0.132 \pm 0.067 \\ 4 & 0.40 \pm 0.06 & -0.03 \pm 0.07 & 0.21 \pm 0.08\end{array}$

a L. Stodolsky and J. Sakurai, Phys. Rev. Letters 11, 90 (1963). b Aachen-Berlin-CERN collaboration, Phys. Letters 22, 533 (1966).

such exchanges were present, one criterion for dipole coupling would be absent. All experiments to date, however, have measured appreciable values for $\rho_{00}$. 
TABLE V. Comparison of experimental vector-meson density matrices in charge-exchange $\pi B \rightarrow V B$ reactions with corresponding $\pi B \rightarrow V \Delta$ reaction.

\begin{tabular}{|c|c|c|c|c|c|}
\hline Reaction & Energy $(\mathrm{GeV} / c)$ & $\rho_{00}$ & $\rho_{1-1}$ & $\operatorname{Re} \rho_{10}$ & Reference \\
\hline$K^{+} n \rightarrow K^{0 *} p$ & 2.3 & $0.60 \pm 0.10$ & $-0.02 \pm 0.08$ & $-0.20 \pm 0.05$ & $a$ \\
\hline$K^{+} p \rightarrow K^{0 *} N^{*++}$ & 3.5 & $0.70 \pm 0.12$ & $0 \pm 0.08$ & $-0.11 \pm 0.07$ & $\mathrm{~b}$ \\
\hline$K^{+} p \rightarrow K^{0 *} N^{*++}$ & 5 & $0.70 \pm 0.2$ & $0.01 \pm 0.08$ & $-0.15 \pm 0.08$ & $\mathrm{~b}$ \\
\hline$\pi^{-} p \rightarrow \rho^{0} n$ & 2.7 & $0.77 \pm 0.1$ & $0.08 \pm 0.07$ & $-0.2 \pm 0.05$ & c \\
\hline$\pi^{+} p \rightarrow \rho^{0} N^{*++}$ & 8 & $0.77 \pm 0.04$ & $-0.035 \pm 0.024$ & $-0.12 \pm 0.03$ & d \\
\hline$\pi^{+} p \rightarrow \rho^{0} N^{*++}$ & 4 & $0.77 \pm 0.04$ & $-0.04 \pm 0.03$ & $-0.06 \pm 0.03$ & $d$ \\
\hline$\pi^{+} n \rightarrow \omega p$ & 1.7 & $0.56 \pm 0.04$ & $-0.05 \pm 0.06$ & $-0.06 \pm 0.04$ & d \\
\hline$\pi^{+} n \rightarrow \omega p$ & 3.25 & $0.62 \pm 0.15$ & $0.19 \pm 0.05$ & $0.25 \pm ?$ & $\mathrm{~d}$ \\
\hline$\pi^{-} p \rightarrow \omega N^{* 0}$ & 2.75 & $0.44 \pm 0.1$ & $0.19 \pm 0.07$ & $-0.05 \pm 0.04$ & $\mathrm{e}$ \\
\hline$\pi^{+} p \rightarrow \omega N^{*++}$ & 2.75 & $0.56 \pm 0.02$ & $0.02 \pm 0.02$ & $-0.02 \pm 0.02$ & e \\
\hline$\pi^{+} p \rightarrow \omega N^{*++}$ & 2.8 & $0.58 \pm 0.04$ & & $-0.10 \pm 0.005$ & $\mathrm{e}$ \\
\hline$\pi^{+} p \rightarrow \omega N^{*++}$ & 4 & $0.47 \pm 0.05$ & $0.13 \pm 0.05$ & $-0.10 \pm 0.03$ & d \\
\hline$\pi^{+} p \rightarrow \omega N^{*++}$ & 8 & $0.26 \pm 0.1$ & $0.17 \pm 0.08$ & $-0.03 \pm 0.06$ & $\mathrm{~d}$ \\
\hline
\end{tabular}

a G. Goldhaber et al., Phys. Rev. Letters 15, 737 (1965).

b R. George et al., CERN Report No. 66-18 (1966) (unpublished).
o D. H. Miller et al., Phys. Rev. 153, 1423 (1967).

calculated error bars for all the reactions, the ratio falls in the range from 0.6 to 0.8 ).

(iii) The $\omega$ production reactions define the ratio $R$ better than the others; they are the ones which tend to limit it to the range $(0.6,0.8)$. This "compensates" for lack of information on $\lim _{t \rightarrow t_{\min } \rho_{00}}$ in these reactions. ${ }^{8}$

These results strongly indicate dipole dominance at the Regge- $N-\Delta$ vertex. However, the $\rho_{00}$ test is weak in the sense that it depends completely on $P=(-1)^{S+1}$ exchanges. ${ }^{7}$ Furthermore, because $P=(-1)^{S}$ exchanges are relatively suppressed at low $t$ in these reactions by kinematic effects, ${ }^{6}$ a conservative interpretation of the data would be that dipole dominance is established only for $P=(-1)^{S+1}$ exchanges.

Fortunately, an independent test of dipole dominance for $P=(-1)^{S}$ exchanges is provided by the reaction $\pi^{+}+p \rightarrow \pi^{0}+\Delta^{++}$, because only $P=(-1)^{S}$ trajectories can couple to the $\pi \pi$ system. The predictions of dipole coupling in our approximate model are compared with experiment in Table IV. It can be seen that the agreement is quite reasonable. Hence, dipole dominance can be accepted as a feature of the Regge $-N-\Delta$ vertex regardless of the parity exchanged.

$$
\pi B \rightarrow V B \text { (Tables I and V) }
$$

Study of the results in Table I shows that little information on possible dominance of dipole or scalar coupling can be obtained from isolated study of the shapes in $t$ of vector-meson density matrix elements. There are two main reasons for this:

(a) Many of the data are concentrated at very small $t$, where the exchange of $P=(-1)^{S+1}$ trajectories is relatively enhanced by kinematic effects. ${ }^{6}$ This parity

${ }^{8}$ At $3-4 \mathrm{GeV} / c, \lim _{t \rightarrow t_{\min } \rho_{00}}=0.5 \pm 0.1$ for the reaction $\pi^{+} p \rightarrow$
$0^{0} \Delta^{++}$(G. Gidal, private communication). This supports the $\omega^{0} \Delta^{++}$(G. Gidal, private communication). This supports the
indication of dipole dominance given by the calculated values of $R$. d Aachen-Berlin-CERN collaboration, Phys. Letters 22, 533 (1966) - Saclay-Orsay-Bari-Bologna collaboration, Phys. Letters 21, 354 (1966). can couple only by dipole coupling, so there is no opportunity for a test.

(b) The density matrix elements predicted by the two couplings for $P=(-1)^{S}$ exchange are so similar that it would be difficult to separate their effects in the natural situation, which is presumably some sort of mixture.

Consideration of the magnitudes of $\rho_{i j}$ in charge exchange $\pi B \rightarrow V B$ reactions does, however, lead to a further test of the Dashen-Frautschi results. This is because charge exchange is synonymous with exchange of an $S U$ (3) octet, given the current experimental set of mesons. The Dashen-Frautschi calculation predicts that non-spin-flip couplings of an octet and nucleon to another nucleon should have $S U(3)$ character $F+\frac{1}{4} D$; this makes the $\rho N N$ and $A_{2} N N$ non-spin-flip couplings relatively small. These are the only well-accepted $P=(-1)^{S}$ trajectories exchanged in the reactions under consideration, and hence the only ones for which nonspin-flip couplings are allowed. As a first approximation, therefore, we might guess that all charge-exchange $\pi B \rightarrow V B$ reactions should be dominated by spin-flip $(J=1)$ couplings. ${ }^{9}$

Octet $J=1$ coupling in the static bootstrap model is predicted to have the remarkable property $g^{B B \theta} / g^{B \Delta \theta}$ $=g^{B B \pi} / g^{B \Delta \pi}$. This means that

$$
f_{\lambda \mu ; 0 \nu}{ }^{s \pi B \rightarrow V B}=\frac{g^{B B \pi}}{g^{B \Delta \pi}} \frac{\left(1 \frac{1}{2} \mu-\nu \nu \mid \frac{1}{2} \mu\right)}{\left(1 \frac{1}{2} \mu-\nu \nu \mid \frac{3}{2} \mu\right)} f_{\lambda \mu ; 0 \nu}{ }^{s \pi B \rightarrow V \Delta}
$$

for corresponding reactions (reactions in which the external particles are the same, save for the $\Delta-B$ replacement), even if several octet trajectories are exchanged. When dipole coupling restrictions are applied to $f^{s} \pi B \rightarrow V \Delta$ and all the vector-meson density matrix

${ }^{9}$ This is consistent with the dominance of spin-flip coupling found in $\pi N$ charge-exchange reactions. 
elements are calculated for both reactions in terms of the parameters $f_{\lambda \mu ; 0 \nu}{ }^{s} \pi B \rightarrow V \Delta$, one discovers that $\rho_{i j}{ }^{\pi B \rightarrow V B}(s, t)$ $=\rho_{i j}{ }^{\pi B \rightarrow V \Delta}(s, t)$ for the vector meson $\rho_{i j}$ 's. Table $\mathrm{V}$ displays the density matrices (averaged over low $t$ ) for several pairs of reactions; the resemblance between the vector-meson density matrices with and without isobar production is quite marked.

We conclude that the predictions of the DashenFrautschi static model bootstrap for $J=1$ and $J=2$ couplings agree in all respects with experiment. At present, it does not seem feasible to test the results for $J=0$ in this fashion.

\section{ACKNOWLEDGMENT}

The author would like to thank Dr. Christoph Schmid for helpful discussions and for reading the manuscript.

\title{
Electroproduction of $\pi$ Mesons at High Energy and Large Momentum Transfer*
}

\author{
RoBert C. VIK† \\ University of California, San Diego, La Jolla, California
}

(Received 17 July 1967)

\begin{abstract}
The inelastic scattering of an electron by a nucleon, accompanied by creation of a single $\pi$ meson (pion electroproduction) is considered as a function of $\mathbf{k}^{\mathbf{2}}$, the square of the three-dimensional momentum transferred from the electron to the final pion-nucleon system. At energies high enough that the pion-nucleon center-of-mass energy is above the region of the $N^{*}(1238)$ resonance, no complete cross-section calculation exists, largely because the presence of inelastic channels makes the calculation difficult. However, the effect of an important set of one-particle-exchange diagrams can be evaluated, and it is proposed that they provide a way of examining the qualitative behavior of the electroproduction cross section as a function of $\mathbf{k}^{2}$. The cross sections in several partial waves, due to these diagrams, are evaluated and are compared with published experimental results at several energies. It is found that the $S_{11}$ and $P_{11}$ partial waves are likely to be important at large values of $\mathbf{k}^{2}$ and at high energies. Furthermore, the dependence of the cross section on $\mathbf{k}^{2}$ in these partial waves is qualitatively like the dependence indicated by the experiments at $1512-\mathrm{MeV}$ pionnucleon center-of-mass energy.
\end{abstract}

\section{INTRODUCTION}

$T^{1}$ HE production of a $\pi$ meson during the inelastic scattering of an electron by a proton (pion electroproduction) will depend not only on the kinematical quantities describing the pion-nucleon final state such as the pion direction of motion and the pionnucleon center-of-mass energy $W$, but also on the momentum transferred to this state from the scattered electron (k). We describe in this paper an estimate of the dependence on $\mathbf{k}^{2}$ of the pion electroproduction cross section at energies above the first pion-nucleon resonance. The difficulties which attend a complete cross-section calculation at high energy are well known. They arise primarily from the presence of the inelastic channels. It seemed possible, however, that a calculation which could be done, namely calculation of the contribution of an important set of one-particleexchange diagrams to the cross section, might reproduce the dependence of the actual cross section on momentum transfer. This is particularly of interest because of the

* Work supported in part by the U. S. Atomic Energy Commission.

$\dagger$ Now at Convair Division of General Dynamics, San Diego, California. data of Cone et al. on electroproduction at large momentum transfer., ${ }^{1,2}$

What is proposed is, in fact, a model that provides a prescription for examing the qualitative behavior of the electroproduction cross section as a function of $\mathbf{k}^{2}$ at high energies. The cross section for production of a pion in each of several pion-nucleon partial waves is calculated and the results are compared with the measurements of Cone $e t a l .{ }^{1}$ The comparison proves to be instructive.

We find, for example, that the $P_{11}$ and $S_{11}$ partial waves are large at large $\mathbf{k}^{2}$ and high energy. They are substantially larger than $D_{13}$ for large $\mathbf{k}^{2}$ and center-of-

${ }^{1}$ A. A. Cone, K. W. Chen, J. R. Dunning, Jr., C. Hartwig, N. F. Ramsey, J. K. Walker, and R. Wilson, Phys. Rev. Letters 14, 326 (1965).

${ }_{2}^{2}$ Throughout the present paper references to Cone et al. mean specifically the preliminary report (Ref. 1). We wish to emphasize, however, that the final report on the experiment has appeared [A. A. Cone, K. W. Chen, J. R. Dunning, Jr., C. Hartwig, N. F. Ramsey, J. K. Walker, and R. Wilson, Phys. Rev. 156, 1490 (1967) ] and gives a more complete listing of the data as well as a phenomenological discussion of the longitudinal contribution to the cross section. The radiative corrections are done more accurately than in the preliminary report with the result that the cross sections are somewhat higher. Nevertheless, the qualitative conclusions of the present paper would be unaltered. 